\title{
Usefulness of Color Doppler Ultrasonography in the Risk Stratification of Thyroid Nodules
}

\author{
Ernesto Maddaloni $^{a}$ b Silvia Irina Briganti ${ }^{a} \quad$ Anna Crescenzi $^{c}$ \\ Giuseppina Beretta Anguissola ${ }^{a}$ Eleonora Perrellac Chiara Taffon ${ }^{c}$ \\ Andrea Palermo ${ }^{a}$ Silvia Manfrini ${ }^{a}$ Paolo Pozzillia Angelo Lauria Pantano ${ }^{a}$ \\ ${ }^{a}$ Endocrinology and Diabetes Unit, Department of Medicine, Campus Bio-Medico University of Rome, \\ Rome, Italy; ${ }^{b}$ Department of Experimental Medicine, Sapienza University of Rome, Rome, Italy; ${ }^{C}$ Pathology Unit, \\ Campus Bio-Medico University of Rome, Rome, Italy
}

\section{Keywords}

Thyroid nodules · Ultrasound · Doppler · Vascularization

\begin{abstract}
Introduction: Thyroid ultrasound (US) is crucial for clinical decision in the management of thyroid nodules. In this crosssectional study, we aimed to test if the evaluation of thyroid nodules' vascularization could improve the risk stratification ability of the American College of Radiology (ACR) TI-RADS classification system. Methods: A total of 873 thyroid nodules undergoing fine-needle aspiration were classified according to ACR TI-RADS US classification. Three types of vascularization were identified: type 0 , no vascular signals; type 1, peripheral vascular signals; type 2, peripheral and intralesional vascular signals. Cytology specimens were evaluated conforming to the Italian Reporting System for Thyroid Cytology, and TIR3b, TIR4, and TIR5 were defined as high risk for malignancy. Odds ratios (ORs) with 95\% confidence intervals $(\mathrm{Cl})$ and the areas under the receiver operating characteristic curves (ROC-AUC) for high-risk cytology categories were calculated. Results: The 3 vascular patterns were differently distributed within the cytology categories: $52.4 \%$ of
\end{abstract}

TIR1C, $15.9 \%$ of TIR2, $5.9 \%$ of TIR3a, $6.7 \%$ of TIR3b, $12.5 \%$ of TIR4, and $28.9 \%$ of TIR5 nodules had no vascular signals ( $p<$ $0.001)$. Nodule vascularity alone was not associated with a higher risk of malignant cytology (OR [95\% Cl] 0.75 [0.431.32], $p=0.32$ ), without differences between peripheral (OR [95\% Cl] 0.65 [0.35-1.20]) and intranodular (OR [95\% Cl] 0.88 [0.48-1.62]) vascularization $(p=0.22)$. The ROC-AUC $(95 \% \mathrm{Cl})$ for the diagnosis of malignant cytology was similar when considering TI-RADS classification alone (0.736 [0.6840.786]) and when considering TI-RADS classification plus the presence/absence of vascular signals (0.736 [0.683-0.789], $p$ value for differences between the ROC-AUCs: 0.91). Among TR1, TR2, and TR3 TI-RADS classes, no nodules without vascular signals showed a malignant cytology, allowing the identification of nodules with benign cytology with $100 \%$ specificity within these US classes. Conclusions: Color Doppler study of thyroid nodules does not improve the risk stratification ability of the ACR TI-RADS US classification system.

(C) 2020 European Thyroid Association Published by S. Karger AG, Basel

E.M. and S.I.B. contributed equally to this work. karger@karger.com

www.karger.com/et

(c) 2020 European Thyroid Association

Published by S. Karger AG, Basel

Karger $\stackrel{2}{=}$

Ernesto Maddaloni, MD, $\mathrm{PhD}$

Department of Experimental Medicine Sapienza University of Rome

Viale Regina Elena 324, IT-00161 Rome (Italy)

ernesto.maddaloni@uniromal.it 


\section{Introduction}

Thyroid nodules affect up to two-thirds of the general population, with $7-15 \%$ being thyroid cancers. The number of thyroid nodules' diagnoses is constantly increasing due to the fortuitous findings during imaging procedures performed for non-thyroidal disorders. Together with the relatively low prevalence of malignant cancers in thyroid nodules, this calls for a correct malignancy risk stratification of the nodules to avoid unnecessary invasive procedures and/or surgery. Thyroid ultrasound (US) examination represents the gold standard for the stratification of thyroid lesions, determining the need for fine-needle aspiration (FNA). An improper use of FNA has the risk of increasing healthcare expenditures and even of inappropriately referring patients to surgery in case of indeterminate cytology [1]. The presence and pattern of nodular vascular signs are often considered among the US features to discriminate benign from malignant nodules $[2,3]$. Despite this widespread credit, there are few data supporting the usefulness of examining thyroid nodules' vascularization for improving the risk stratification of thyroid nodules $[4,5]$. In a previous study, we showed that the TI-RADS US classification system developed by the American College of Radiology (ACR) in 2017 has a better performance in discriminating nodules that are more likely to have high-risk cytological results compared to other US classification systems [6]. However, the ACR TI-RADS system does not consider color Doppler signs because of its unclear value in differentiating cancer from benign nodules $[7,8]$. In the present study, we aim to test whether the evaluation of thyroid nodules' vascularization can improve the risk stratification ability of the ACR TI-RADS classification system.

\section{Subjects and Methods}

The protocol of this cross-sectional study has been previously fully described [6]. Briefly, data about US features and vascularity of all nodules undergoing FNA from January 2015 to May 2016 at the Unit of Endocrinology and Diabetes of Campus Bio-Medico University of Rome were retrieved from medical records. Thyroid US with real-time color Doppler was performed at a frequency range of $10-12 \mathrm{MHz}$ on a MyLab 50 (Esaote, Genova, Italy). As this is an observational real-world study, nodules were triaged for biopsy by clinicians in charge of the patients. Therefore, all FNAs were performed based on an impartial clinical indication, independent from the study. For this analysis, we retrospectively classified nodules according to ACR TI-RADS risk stratification criteria as follows: based on the description retrieved from medical records, a yes or no answer for each of the features derived from the ACR guidelines about composition, echogenicity, shape, mar- gin, and echogenic foci was chosen by an investigator blinded to cytological results. Scores and categories were then calculated according to the ACR guidelines [8]. The vascularity pattern, in detail, was evaluated using sagittal and transverse scans according to the classification system proposed by Papini et al. [9] which includes 3 types of vascularity: type 0 , no vascular signals; type 1 , peripheral vascular signals; type 2, peripheral and intralesional vascular signals. Cytology specimens were evaluated by expert cytopathologists at the Unit of Pathology conforming to Italian Reporting System for Thyroid Cytology [10] as follows: TIR1 (non-diagnostic), TIR1c (non-diagnostic/cystic), TIR2 (non-malignant/benign), TIR3a (low-risk indeterminate lesion), TIR3b (high-risk indeterminate lesion), TIR4 (suspicious of malignancy), or TIR5 (malignant). Nodules with TIR1 cytology were excluded from the study. Nodules with TIR1c cytology were included in the study and were considered clinically non-malignant/benign. Cyst fluid may yield only macrophages, but the risk of malignancy is low for these lesions if they are simple and under $3 \mathrm{~cm}$. For this reason, these cases are separated from inadequate specimens obtained from solid lesions and are reported as nondiagnostic followed by the subcategory "cyst fluid only." In the proper clinical setting (e.g., US evidence of a simple, unilocular cyst), these specimens may be considered clinically adequate, even though they are reported as non-diagnostic [11]. In case of disagreement, definitive reporting was achieved by mutual consensus. A preliminary analysis of 251 nodules with definitive pathology diagnosis served as an internal validation to confirm the significant risk of malignancy associated with TIR3b, TIR4, and TIR5 categories (prevalence of cancer within each category: $68.2 \%, 82.4 \%$, and $98.3 \%$, respectively) and has already previously been reported [6].

\section{Statistical Analysis}

Values are expressed as means \pm standard deviation (SD) or medians (25-75th percentiles) for continuous variables and as proportions for categorical variables. Shapiro-Wilk normality test was used to assess the normality of continuous variables' distributions (variables with Shapiro-Wilk statistic $<0.9$; $p$ values $<0.05$ were considered non-normally distributed). Groups were compared by analysis of variance, Kruskal-Wallis, $\chi^{2}$, and Fisher's exact test depending on distribution. Binary logistic regression was used to evaluate the odds ratios (OR) of malignant cytology based on 1 or more predictors, and the areas under the receiving operator characteristic (ROC) curves (AUC), or C-index, of regression models were tested for equality [12]. In particular, to test whether the information about vascular patterns improves the diagnostic accuracy of TI-RADS, the ROC-AUC of logistic regression models having TI-RADS classes alone or both TIRADS classes and vascular patterns as predictors were compared. Non-parametric variables were natural log-transformed before testing in the model. As previously reported, the binary dependent variable "high-risk cytology for malignancy" was defined for cytological categories TIR3b, TIR4, and TIR5, while cytological categories TIR1c, TIR2, and TIR3a identified nodules with lowrisk cytology. Sensitivity and specificity of TR1, TR2, and TR3 US TI-RADS classes for low-risk cytology and of TR4 and TR5 classes for high-risk cytology, with and without information about vascularization, were calculated. Statistical significance was set as $p<0.05$. Stata/IC 12.1 software (StataCorp) was used for data analysis. 
Table 1. Distribution of the color Doppler ultrasonographic patterns within the different cytology categories

\begin{tabular}{|c|c|c|c|c|c|c|c|}
\hline $\begin{array}{l}\text { Vascular } \\
\text { pattern }\end{array}$ & $\begin{array}{l}\text { TIR1c } \\
\text { (size 19 [11-36]) }\end{array}$ & $\begin{array}{l}\text { TIR2 } \\
\text { (size 14 [10-20]) }\end{array}$ & $\begin{array}{l}\text { TIR3a } \\
\text { (size 16 [10-21]) }\end{array}$ & $\begin{array}{l}\text { TIR3b } \\
\text { (size } 13 \text { [9-17]) }\end{array}$ & $\begin{array}{l}\text { TIR4 } \\
\text { (size } 10[7-18] \text { ) }\end{array}$ & $\begin{array}{l}\text { TIR5 } \\
\text { (size } 9 \text { [7-12]) }\end{array}$ & Total \\
\hline 0 & $11(52.4)$ & $94(15.9)$ & $10(5.8)$ & $2(6.6)$ & $2(12.5)$ & $13(28.8)$ & 132 \\
\hline 1 & $7(33.3)$ & $291(49.3)$ & $76(44.4)$ & $13(43.3)$ & $6(37.5)$ & $17(37.7)$ & 410 \\
\hline 2 & $3(14.2)$ & $205(34.7)$ & 85 (49.7) & $15(50)$ & $8(50)$ & $15(33.3)$ & 331 \\
\hline
\end{tabular}

Size of nodules in the largest diameter within each category is reported in millimeters as median [25-75th percentile].

Table 2. Distribution of the color Doppler ultrasonographic patterns within the different ultrasonographic TI-RADS categories

\begin{tabular}{lllllll}
\hline $\begin{array}{l}\text { Vascular } \\
\text { classification }\end{array}$ & \multicolumn{2}{l}{ TI-RADS classification, $n(\%)$} & Total \\
\cline { 2 - 6 } & 1 & 2 & 3 & \multicolumn{2}{l}{4} & \\
\hline 0 & $24(43.6)$ & $19(10.8)$ & $10(6.6)$ & $59(14.4)$ & $20(23.8)$ & 132 \\
1 & $21(38.2)$ & $71(40.6)$ & $68(45.3)$ & $214(52.3)$ & $36(42.9)$ & 410 \\
2 & $10(18.2)$ & $85(48.6)$ & $72(48)$ & $136(33.3)$ & $28(33.3)$ & 331 \\
\hline Total & 55 & 175 & 150 & 409 & 84 & 873 \\
\hline
\end{tabular}

\section{Results}

\section{Population and Nodules' Features}

A total of 1,170 nodules undergoing FNA were screened for entering in the study. Of these, 218 had no information about vascularization and were excluded from this analysis. Of the remaining 952 nodules, 79 had a non-diagnostic cytology and were also excluded. Therefore, 873 nodules (size in the largest diameter: 14 [10-20] $\mathrm{mm}$ ) from 739 patients ( $80 \%$ females; mean age: $55.3 \pm$ 13.4 years) were included in the analysis, 132 (15.1\%) showing no vascular signs, $410(47.0 \%)$ showing the vascular pattern 1, and 331 (37.9\%) showing the vascular pattern 2 . Nodules showing no vascular signs were smaller in the largest diameter $(11[8-16] \mathrm{mm})$ compared to nodules showing both peripheral $(14[10-20] \mathrm{mm}, p<$ $0.001)$ and peripheral and intralesional vascular patterns (15 [10-22] mm, $p<0.001)$, with no difference in nodule size between the two vascular pattern categories $(p=$ $0.102)$. Ninety-one nodules (10.4\%) had a cytology diagnosis within the high-risk for malignancy categories TIR3b, TIR4, and TIR5 (see online suppl. Table S1 [see www.karger.com/doi/10.1159/000509325 for all online suppl. material] for the cytology categories and online suppl. Table S2 for TI-RADS classes of the 873 nodules). The three vascular patterns were differently distributed within the cytology categories. Specifically, $52.4 \%$ of TIR1c, $15.9 \%$ of TIR2, $5.9 \%$ of TIR3a, $6.7 \%$ of TIR3b, $12.5 \%$ of TIR4, and $28.9 \%$ of TIR 5 nodules had no vascular signs $(p<0.001$; Table 1$)$. Similarly, vascular patterns were also differently distributed within the different ultrasonographic TI-RADS categories $(p<0.001$; Table 2).

\section{Vascularization Features and Risk of Malignant Cytology}

In the entire sample, the presence of vascular signals was not associated with a higher risk of malignant cytology (OR 0.75 [0.43-1.32], $p=0.32$ ), without differences between peripheral (OR [95\% CI] $0.65[0.35-1.20])$ and intranodular (OR [95\% CI] 0.88 [0.48-1.62]) vascularization ( $p$ value for difference of regression coefficients: 0.22 ). These results did not significantly change after adjusting for nodule size. Accordingly, the ROC-AUC (95\% CI) for the diagnosis of malignant cytology was similar when considering the TI-RADS classification alone $(0.736$ [0.684-0.786]) and when considering the TI-RADS classification plus the presence/absence of vascular signs 
Table 3. Sensitivity and specificity for low-risk cytology of the low-risk ultrasonographic TI-RADS classes

\begin{tabular}{|c|c|c|c|c|c|}
\hline \multirow{2}{*}{$\begin{array}{l}\text { TI-RADS } \\
\text { classes }\end{array}$} & \multicolumn{2}{|c|}{ Not considering vascularization } & \multicolumn{2}{|c|}{ Considering vascularization } & \multirow[t]{2}{*}{$p$ value } \\
\hline & sensitivity, $\%$ & specificity, \% & sensitivity, \% & specificity, \% & \\
\hline TR1 & 6.78 & 97.8 & 3.07 & 100 & \\
\hline TR2 & 22.00 & 96.7 & 2.40 & 100 & \\
\hline TR3 & 17.80 & 87.9 & 1.28 & 100 & \\
\hline AUC-ROC & \multicolumn{2}{|c|}{0.644} & \multicolumn{2}{|c|}{$\mathrm{N} / \mathrm{A}$} & N/A \\
\hline
\end{tabular}

Table 4. Sensitivity and specificity for high-risk cytology of the high-risk ultrasonographic TI-RADS classes

\begin{tabular}{|c|c|c|c|c|c|}
\hline \multirow{2}{*}{$\begin{array}{l}\text { TI-RADS } \\
\text { classes }\end{array}$} & \multicolumn{2}{|c|}{ Not considering vascularization } & \multicolumn{2}{|c|}{ Considering vascularization } & \multirow[t]{2}{*}{$p$ value } \\
\hline & sensitivity, \% & specificity, \% & sensitivity, \% & specificity, \% & \\
\hline TR4 & 44.0 & 52.8 & 37.4 & 59.6 & \\
\hline TR5 & 38.5 & 93.7 & 26.4 & 94.9 & \\
\hline AUC-ROC & \multicolumn{2}{|l|}{0.645} & \multicolumn{2}{|c|}{0.591} & 0.011 \\
\hline
\end{tabular}

(0.736 [0.683-0.789], $p$ for differences between the ROCAUC 0.909).

Table 3 shows the sensitivity and specificity for benign cytology of TR1, TR2, and TR3 US TI-RADS classes and Table 4 for malignant cytology of TR4 and TR5 classes, with and without information about vascularization. No nodules without vascular signs within US classes TR1, TR2, and TR3 showed a malignant cytology. Therefore, the information about the absence of vascularization in nodules within TR1, TR2, or TR3 classes allowed the identification of nodules with benign cytology with $100 \%$ specificity. The improvement of specificity was particularly relevant for nodules within the TR class 3, moving from 87.9 to $100 \%$. On the contrary, the presence of vascular signs in nodules within the TR classes 4 and 5 only slightly improved the specificity for malignancy (from 52.9 to $59.6 \%$ and from 93.7 to $94.9 \%$ for TR4 and TR5 classes, respectively), at the expense of a relevant reduction in sensitivity (from 44 to $37.4 \%$ and from 38.5 to $26.4 \%$, respectively). This resulted in a ROC-AUC of 0.645 for the identification of nodules with high-risk cytology for the TR4/TR5 test (being within the TR4 or the TR5 classes) and of 0.591 for the TR4/TR5 plus vascular sign test (being within the TR4 or TR5 classes and showing vascular signs at the Doppler examination; $p$ value for ROC-AUC difference $=0.011)$.

\section{Discussion}

This study shows that information about a vascular signal does not improve the overall accuracy of the ACR TI-RADS US classification for thyroid nodules risk stratification. In particular, the presence of a vascular signal was not associated with a significant increase in the risk of malignant cytology. This may be due to US technology development with new instruments detecting blood flow imaging of vessels with very small diameter, therefore increasing the prevalence of vascularized nodules, as confirmed in our records where the great majority (84.9\%) of nodules shows signs of vascularization.

Our data contribute to a debated topic, as different previous studies showed controversial results in terms of the predictive value for malignancy of nodule vascularization. Some studies showed that thyroid cancers more frequently have a central and peripheral vascularization on Doppler US, while benign nodules less frequently present vascular signs or present a mild peripheral blood flow $[13,14]$. This was also suggested in a meta-analysis by Brito et al. [15], showing that the odds of having intranodular blood flow in cancers are significantly higher than the odds of having such a sign in benign nodules (diagnostic OR 1.8 [1.5-2.2]), even though other US features showed higher diagnostic accuracy. Another metaanalysis including a total of 1,048 nodules with available 
data about color Doppler signs showed that nodules with intranodular blood flow are 4 times more likely of being cancers than nodules without intranodular blood flow [16]. However, Khadra et al. [17] showed in another recent meta-analysis that an enhanced vascularization on Doppler sonography is not predictive of malignancy in all nodules, but may help in the risk stratification of nodules with suspicious US features. On the contrary, we found that information about vascularity specifically in nodules with high-suspicion US features (TR4 and TR5) significantly reduced the ROC-AUC of ACR TI-RADS for the identification of malignant nodules, due to a significant reduction of its sensitivity. Of note, our study includes a large sample of 873 nodules in a single study and from a single center, without risk of heterogeneity in vascularity description and cytological assessment.

Our data agree with previous findings from Shin et al. [18], who surprisingly showed that nodules with central vascularization were more frequently benign compared to avascular nodules, also after adjustment for nodule dimension. Other recent studies also showed that more than 50\% of nodules with an increased vascularization were histologically benign and that avascular nodules with suspicious characteristics may hide thyroid cancers $[4,5]$. Accordingly, we found that $18.7 \%$ of nodules with high-risk cytology had no vascular signals (vascular pattern 0 ) and that $37.5 \%$ of nodules with low-risk cytology had intralesional vascular signs (vascular pattern 2). It has been hypothesized that the contrasting results between different studies and the different proportion of avascular malignant nodules in some studies, such as ours, may be due to a different relative prevalence of papillary and follicular neoplasms $[5,19]$. While follicular thyroid cancers are characterized by a rich intranodular vascularization, papillary thyroid cancers are rich in lymphatic vessels, but poorer in blood vessels when compared to follicular cancers [20].

Our cross-sectional analysis also suggests that there may be little benefit in the description of nodule vascularization for nodules belonging to TR1, TR2, and TR3 US TI-RADS classes. In the absence of vascular signs, nodules within these classes never showed a high-risk cytology, and further evaluation may, therefore, be avoided. However, these nodules represent only a small percentage ( $6 \%$ in our selected records) of all thyroid nodules.

While we showed that traditional evaluation of nodule vascularization is of limited value, improvements in the risk stratification may be achieved by next-generation US machines. It is reasonable to suppose that future studies will be based on data obtained from 3-dimensional pow-

Doppler Ultrasonography in the

Stratification of Thyroid Nodules er Doppler ultrasound (PDUS) imaging and 3-dimensional contrast-enhancement ultrasound (CEUS) to detect nodules at major risk to be thyroid cancers. These techniques can evaluate different US characteristics at different levels of spatial resolution. CEUS can distinguish macro- and microvasculature of thyroid nodules thanks to the backscatter of the US waves produced after the injection of a contrast composed of microbubbles. PDUS, on the contrary, can identify the precise amount of blood flow in well-formed blood vessels, even if with poor resolution. The use of these new techniques could give additional information about the quality of nodular vascularization. Blood vessels with an irregular or turbulent course could be the consequence of a chaotic angiogenetic process which is generally associated with malignant proliferation [21].

We acknowledge that our study is limited by a possible selection bias due to the evaluation of nodules already selected for FNA and by the absence of data about thyroid function tests and/or thyroid scintigraphy. For this reason we cannot exclude the presence of functioning adenomas among the nodules we included in the analysis, especially among nodules within the TIR3a category and with intranodular vascular signs. Furthermore, the final diagnoses were based on the cytopathology and not on surgical histology, which may cause false-negative and false-positive results. However, the probability of a false diagnosis in TIR2 and TIR5 categories is low at $<3 \%$ and $<1 \%$, respectively [10], as compared to histopathology, and we previously confirmed the significant risk of malignancy associated with TIR3b, TIR4, and TIR5 categories in our sample [6]. Strengths of this study include the large sample size and the homogenous evaluation of all US, color Doppler, and cytological features from a single high-volume thyroid center.

In conclusion, our study shows that the color Doppler study of thyroid nodules for the evaluation of their vascularization does not improve the risk stratification ability of the ACR TI-RADS 2017 US classification system, with only little benefit associated with the exclusion of the risk of malignancy in the minority of nodules without both suspicious US features and vascular signs.

\section{Statement of Ethics}

The study was conducted in accordance with the principles of the Declaration of Helsinki and was approved by the Ethics Committee of Campus Bio-Medico University, Rome, Italy (16.16PT). All subjects gave written consent for the use of their anonymized data for research purposes.

Eur Thyroid J 2021;10:339-344

DOI: $10.1159 / 000509325$ 


\section{Conflict of Interest Statement}

The authors have nothing to disclose.

\section{Funding Source}

There is no funding to declare.

\section{References}

1 Anil G, Hegde A, Chong FH. Thyroid nodules: risk stratification for malignancy with ultrasound and guided biopsy. Cancer Imaging. 2011 Dec;11(1):209-23.

2 Phuttharak W, Somboonporn C, Hongdomnern G. Diagnostic performance of gray-scale versus combined gray-scale with colour doppler ultrasonography in the diagnosis of malignancy in thyroid nodules. Asian Pac J Cancer Prev. 2009;10(5):759-64.

3 Iared W, Shigueoka DC, Cristófoli JC, Andriolo R, Atallah AN, Ajzen SA, et al. Use of color Doppler ultrasonography for the prediction of malignancy in follicular thyroid neoplasms: systematic review and meta-analysis J Ultrasound Med. 2010 Mar;29(3):419-25.

4 Varverakis E, Neonakis E, Tzardi M, Chrysos E. Role of color Doppler ultrasonography in the preoperative management of cold thyroid nodules. Hormones (Athens). 2007 Jan-Mar; 6(1):44-51.

5 Yang GC, Fried KO. Most Thyroid Cancers Detected by Sonography Lack Intranodular Vascularity on Color Doppler Imaging: Review of the Literature and SonographicPathologic Correlations for 698 Thyroid Neoplasms. J Ultrasound Med. 2017 Jan;36(1): 89-94.

6 Lauria Pantano A, Maddaloni E, Briganti SI, Beretta Anguissola G, Perrella E, Taffon C, et al. Differences between ATA, AACE/ACE/ AME and ACR TI-RADS ultrasound classifications performance in identifying cytological high-risk thyroid nodules. Eur J Endocrinol. 2018 Jun;178(6):595-603.

7 Grant EG, Tessler FN, Hoang JK, Langer JE, Beland MD, Berland LL, et al. Thyroid Ultrasound Reporting Lexicon: White Paper of the ACR Thyroid Imaging, Reporting and Data System (TIRADS) Committee. J Am Coll Radiol. 2015 Dec;12(12 Pt A):1272-9.

\section{Author Contributions}

E.M. designed the study, analyzed and interpreted the data, and wrote the manuscript. S.I.B. retrieved the data, interpreted the results, and wrote the manuscript. A.C., E.P., and C.T. evaluated the cytology specimens and critically reviewed the manuscript. G.B.A. and A.P. performed FNA and critically reviewed the manuscript. S.M. and P.P. helped with data interpretation and critically reviewed the manuscript. A.L.P. designed the study, retrieved and interpreted the data, and critically reviewed the manuscript.
8 Tessler FN, Middleton WD, Grant EG, Hoang JK, Berland LL, Teefey SA, et al. ACR Thyroid Imaging, Reporting and Data System (TIRADS): White Paper of the ACR TI-RADS Committee. J Am Coll Radiol. 2017 May; 14(5):587-95.

9 Papini E, Guglielmi R, Bianchini A, Crescenzi A, Taccogna S, Nardi F, et al. Risk of malignancy in nonpalpable thyroid nodules: predictive value of ultrasound and color-Doppler features. J Clin Endocrinol Metab. 2002 May; 87(5):1941-6.

10 Nardi F, Basolo F, Crescenzi A, Fadda G, Frasoldati A, Orlandi F, et al. Italian consensus for the classification and reporting of thyroid cytology. J Endocrinol Invest. 2014 Jun;37(6): 593-9.

11 Ali SZ, Cibas ES, editors. The Bethesda system for reporting thyroid cytopathology: definitions, criteria, and explanatory notes. Springer; 2018.

12 DeLong ER, DeLong DM, Clarke-Pearson DL. Comparing the areas under two or more correlated receiver operating characteristic curves: a nonparametric approach. Biometrics. 1988 Sep;44(3):837-45.

13 Chammas MC, Gerhard R, de Oliveira IR, Widman A, de Barros N, Durazzo M, et al. Thyroid nodules: evaluation with power Doppler and duplex Doppler ultrasound. Otolaryngol Head Neck Surg. 2005 Jun;132(6):87482.

14 Sultan LR, Xiong H, Zafar HM, Schultz SM, Langer JE, Sehgal CM. Vascularity assessment of thyroid nodules by quantitative color Doppler ultrasound. Ultrasound Med Biol. 2015 May;41(5):1287-93.

15 Brito JP, Gionfriddo MR, Al Nofal A, Boehmer KR, Leppin AL, Reading C, et al. The accuracy of thyroid nodule ultrasound to predict thyroid cancer: systematic review and metaanalysis. J Clin Endocrinol Metab. 2014 Apr; 99(4):1253-63.
16 Woliński K, Szkudlarek M, Szczepanek-Parulska E, Ruchała M. Usefulness of different ultrasound features of malignancy in predicting the type of thyroid lesions: a meta-analysis of prospective studies. Pol Arch Med Wewn. 2014;124(3):97-104.

17 Khadra H, Bakeer M, Hauch A, Hu T, Kandil E. Is vascular flow a predictor of malignant thyroid nodules? A meta-analysis. Gland Surg. 2016 Dec;5(6):576-82.

18 Shin JH, Baek JH, Chung J, Ha EJ, Kim JH, Lee YH, et al.; Korean Society of Thyroid Radiology (KSThR) and Korean Society of Radiology. Ultrasonography Diagnosis and Imaging-Based Management of Thyroid Nodules: Revised Korean Society of Thyroid Radiology Consensus Statement and Recommendations. Korean J Radiol. 2016 May-Jun;17(3): 370-95.

19 Haugen BR, Alexander EK, Bible KC, Doherty GM, Mandel SJ, Nikiforov YE, et al. 2015 American Thyroid Association Management Guidelines for Adult Patients with Thyroid Nodules and Differentiated Thyroid Cancer: The American Thyroid Association Guidelines Task Force on Thyroid Nodules and Differentiated Thyroid Cancer. Thyroid. 2016 Jan;26(1):1-133.

20 Giorgadze TA, Baloch ZW, Pasha T, Zhang PJ, Livolsi VA. Lymphatic and blood vessel density in the follicular patterned lesions of thyroid. Mod Pathol. 2005 Nov;18(11):142431.

21 Caresio C, Caballo M, Deandrea M, Garberoglio R, Mormile A, Rossetto R, et al. Quantitative analysis of thyroid tumors vascularity: A comparison between 3-D contrastenhanced ultrasound and 3-D Power Doppler on benign and malignant thyroid nodules. Med Phys. 2018 Jul;45(7):3173-84. 